\title{
AN ECONOMIC STUDY OF THE MOST IMPORTANT OIL CROPS IN EGYPT
}

Fawzia A. Saber ; AZZA M. Ghazala and Samar M. Alkady

Department of Economic Studies - Division of Economic and Social Studies - Desert search Center - Cairo - Egypt.

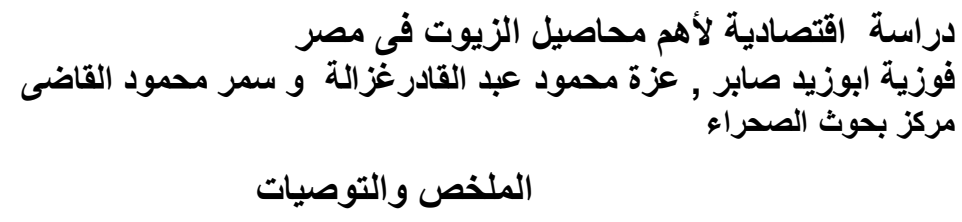

تعتبر المحاصيل الزيتية من المحاصيل الاستر اتيجية الهامة سواء فى مصر او فى دول العالم وذلك لأنها تمثل

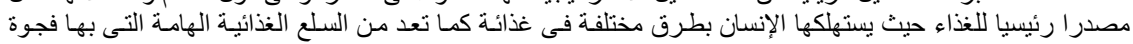

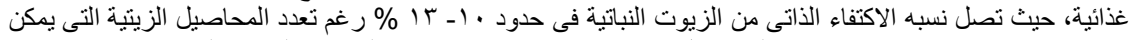

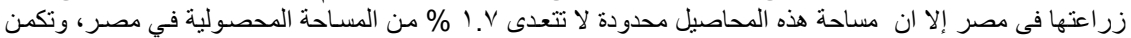

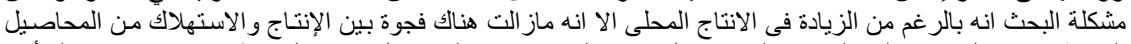

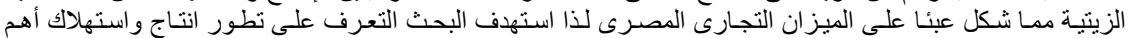

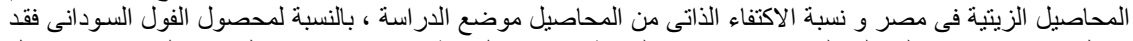

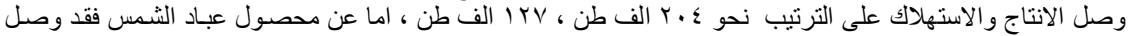

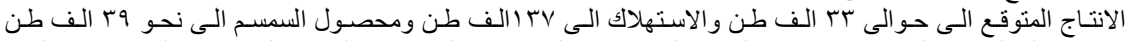

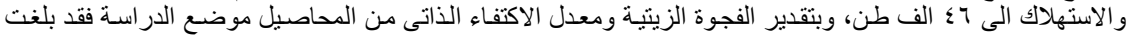

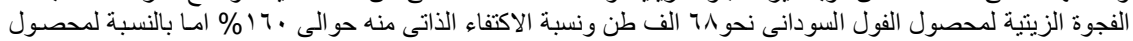

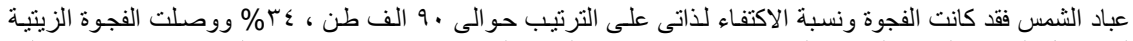

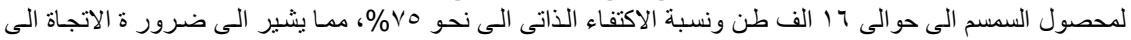

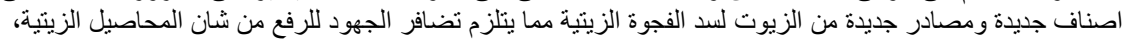

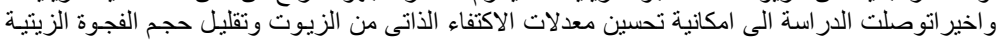

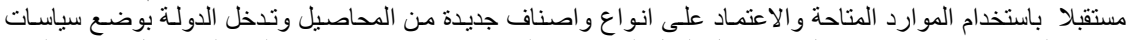

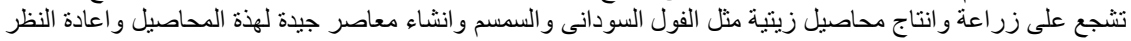

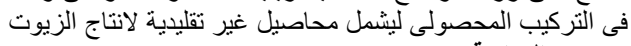
وتوصى الدراسة التركية

ا - وضع استرتيجية واهداف للنهوض بالمحاصيل الزيتية تضم زر اعة محاصيل زيتية جديدة يعتمد عليها فى سد الفجوة الزيتية .

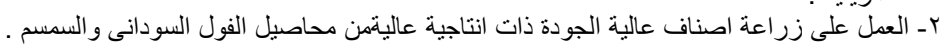

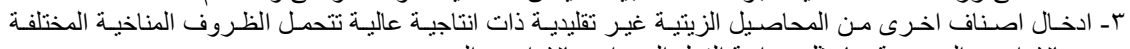

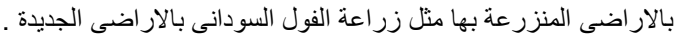

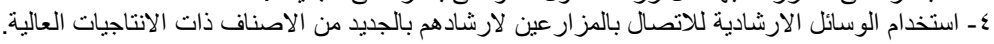

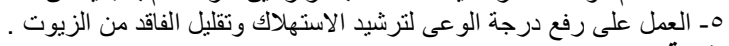

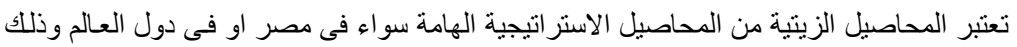
مقدمة : مقل

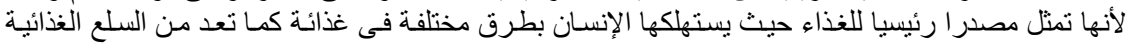

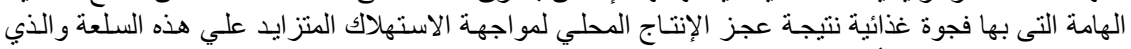

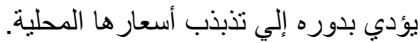

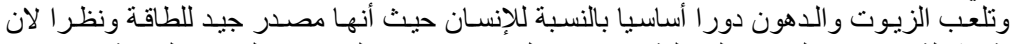

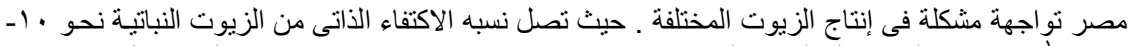

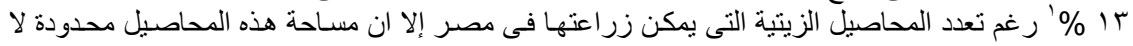




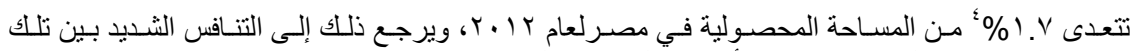

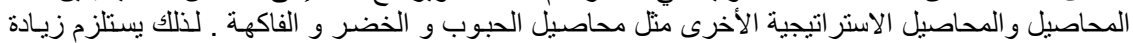

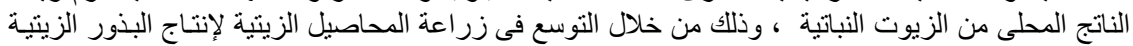

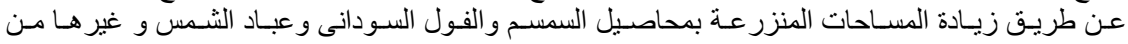

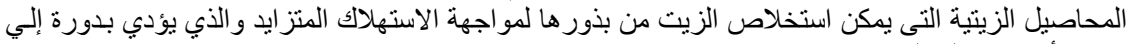
تذبذب أسعار ها المحلية . مشكلة الار اسعة:

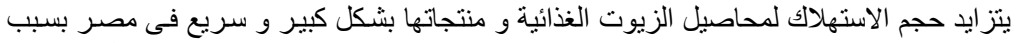

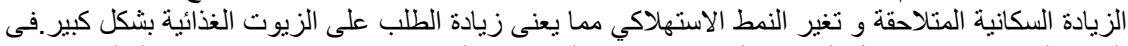

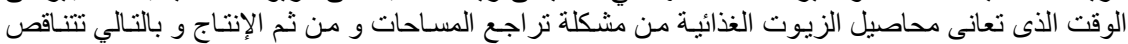

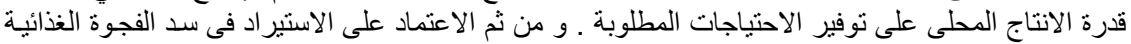

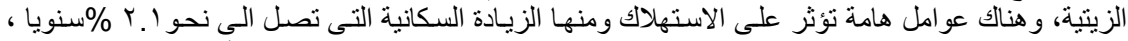

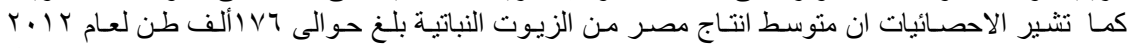

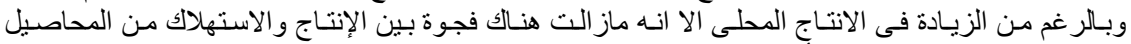

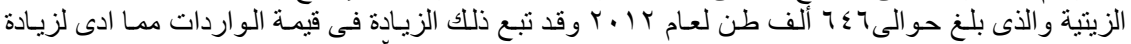

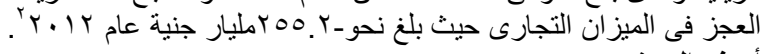

حيث ان محاصيل الزيوت الغذائية و منتجاتها من الزيوت تعد واحدة من اهم السـلع الاستر اتيجية

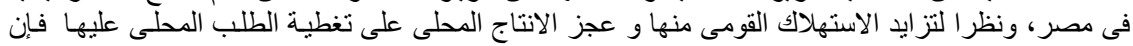

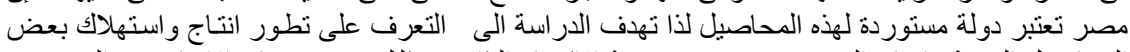

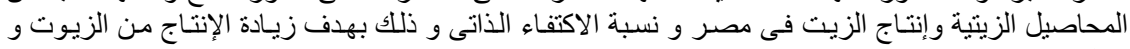

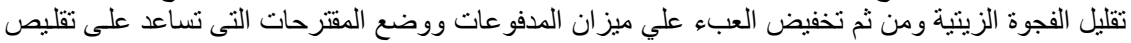
حجم الفجوة الغذائية الزيتية وتحسين نسب الفئ الاكتفاء الذاتى .

\section{الطريقة البحثية ومصادر البيانات}

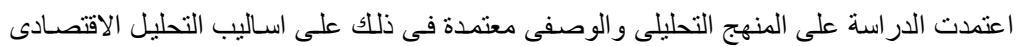

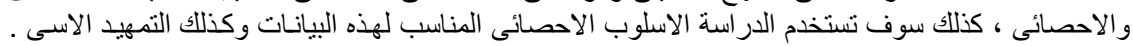

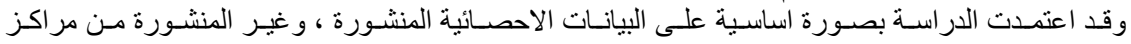

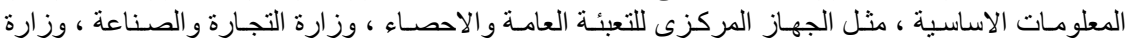

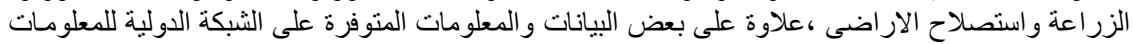

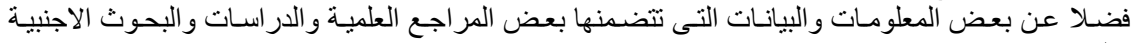

\section{مناقشة النتائج}

و المصرية .

إنتاج المحاصيل الزيتية فى مصر :

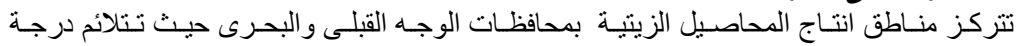

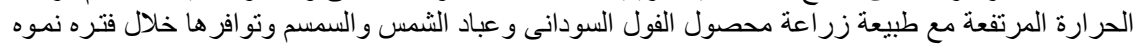

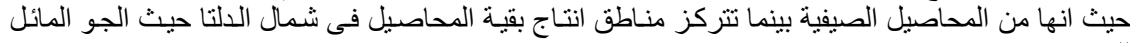




\section{أولاً: المؤشرات الانتاجية للمحاصيل موضع الاراسة :

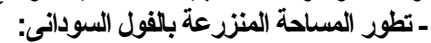

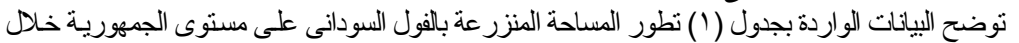

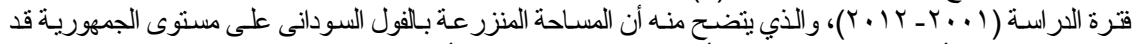

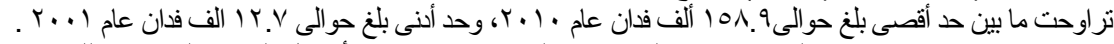

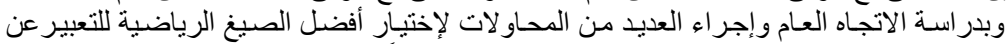

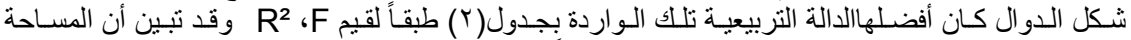

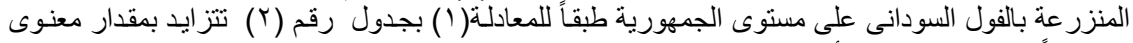

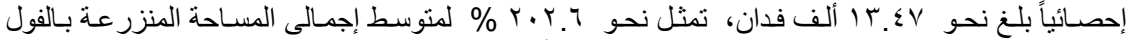

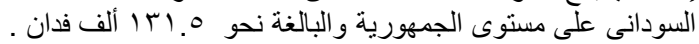

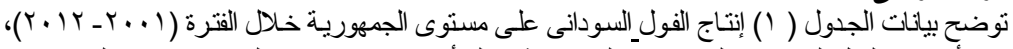

تطور إنتاج القول السودانى:

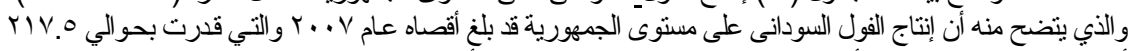

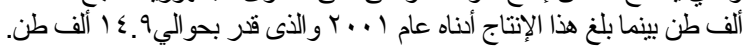

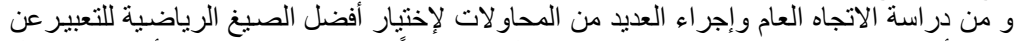

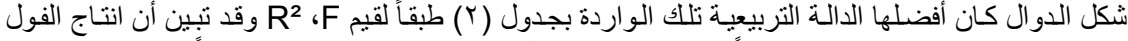

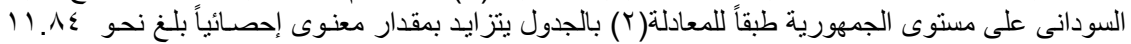

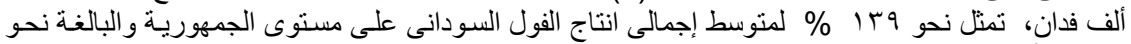

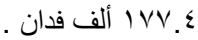

ـ تطور المساحة المنزرعة بعباد الثمس:

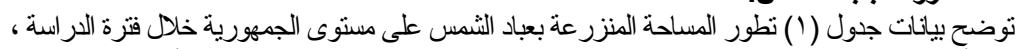

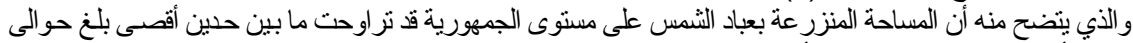

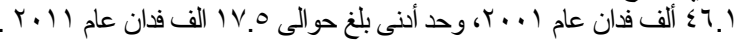

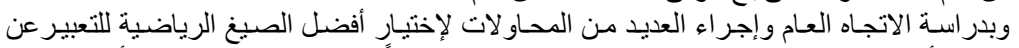

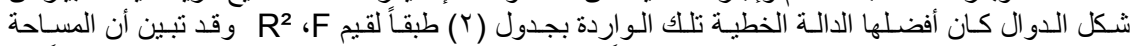

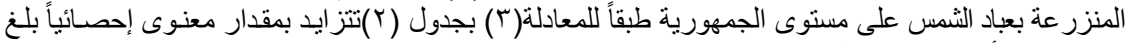

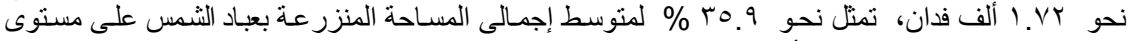

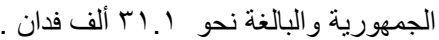
جدول (1): اجمالى مساحة وانتاج وانتاجية محصول الفول السودانى وعباد الثمس والسمسم بجمهوريـة

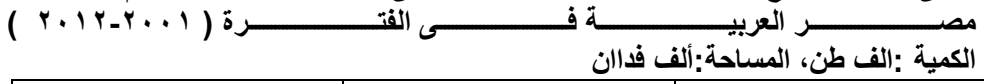

\begin{tabular}{|c|c|c|c|c|c|c|c|c|c|}
\hline \multicolumn{3}{|c|}{ السمسم } & \multicolumn{3}{|c|}{ عباد الثمس } & \multicolumn{3}{|c|}{ الفول السو دانى } & \multirow{2}{*}{ السنوت } \\
\hline انتاج & انتاجية & مساحة & انتاج & انتاجية & مساحة & انتاج & انتاجية & مساحة & \\
\hline 34.8 & 0.513 & 67.9 & 44.1 & 0.958 & 46.1 & 14.9 & 1.173 & 12.7 & $r \ldots 1$ \\
\hline 36.8 & 0.51 & 72.1 & 35.0 & 0.95 & 33.3 & 191.0 & 1.354 & 141.1 & $r \ldots r$ \\
\hline 36.7 & 0.513 & 71.5 & .636 & 0.976 & 32.4 & 110.3 & 1.308 & 84.3 & $r \ldots r$ \\
\hline 36.9 & 0.531 & 69.6 & 43.3 & 0.974 & 45.5 & 191.4 & 1.329 & 144.0 & $r \ldots \varepsilon$ \\
\hline 36.7 & 0.549 & 66.9 & 30.4 & 0.967 & 31.5 & 199.3 & 1.347 & 148.0 & $r \ldots o$ \\
\hline 40.6 & 0.554 & 73.4 & 35.8 & 1.004 & 35.6 & 183.9 & 1.392 & 132.1 & $r \ldots T$ \\
\hline 41.5 & 0.555 & 74.9 & 37.6 & 1.017 & 27.2 & 217.5 & 1.401 & 155.3 & $r \ldots V$ \\
\hline 36.5 & 0.55 & 66.4 & 39.4 & 1.1 & 19.3 & 208.8 & 1.4 & 146.2 & $r \cdots \lambda$ \\
\hline 50.0 & 0.5 & 98.8 & 35.6 & 0.99 & 39.6 & 198.0 & 1.3 & 151.9 & $r \ldots q$ \\
\hline 46.2 & 0.53 & 74.9 & 36.8 & 1.1 & 35.3 & 202.0 & 1.3 & 158.9 & $r \cdot 1 \cdot$ \\
\hline 43.2 & 0.552 & 78.3 & 18.3 & 1.045 & 17.5 & 206.5 & 1.334 & 154.8 & $r .11$ \\
\hline 31.3 & 0.54 & 57.6 & 20.0 & 1.1 & 17.7 & 205.4 & 1.4 & 148.7 & $r . I r$ \\
\hline 39.3 & 0.53 & 67.1 & 61.5 & 1.01 & 31.1 & 177.4 & 1.3 & 131.5 & المتوسط \\
\hline
\end{tabular}




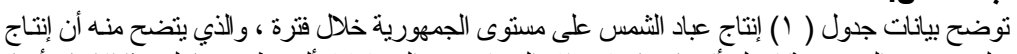

تطور إنتاج عباد الثنمس:

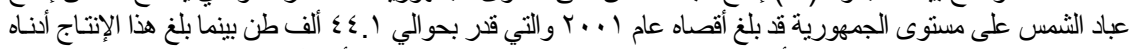

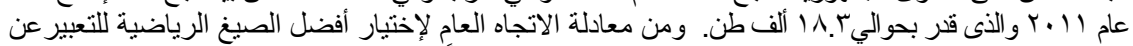

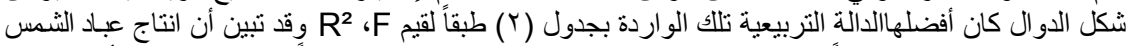

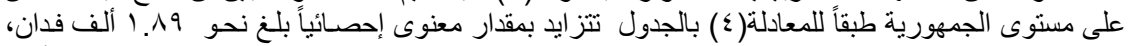

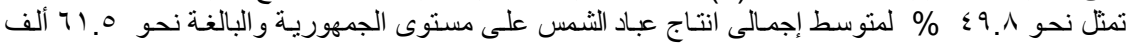

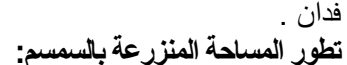

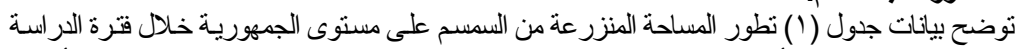

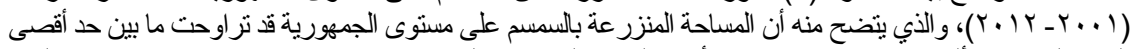

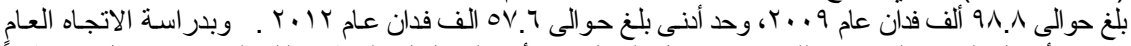

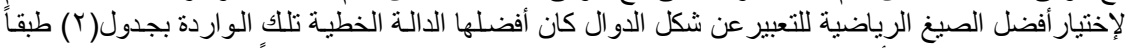

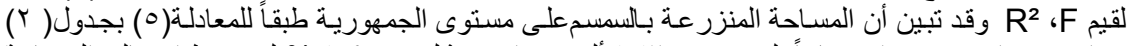

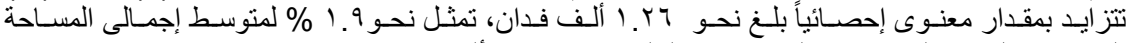

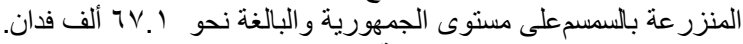

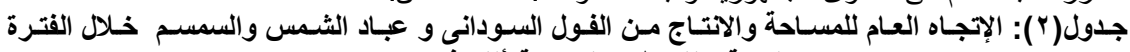

\begin{tabular}{|c|c|c|c|c|c|c|c|c|}
\hline $\begin{array}{l}\text { معدل التغير \% } \\
\text { السنوى }\end{array}$ & المتوسط & ف & is & المعادلة & التقابعير & | & المعادلة & |المحصول| \\
\hline$r \cdot T . T$ & $1+1.0$ & ¿. $V$ & זיד & 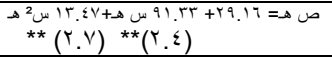 & المساحة & |تربيعية & 1 & \multirow[t]{2}{*}{ الفول السودانى } \\
\hline 149 & IVY. $\varepsilon$ & 7.9 & VY & 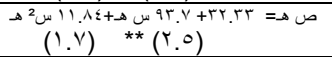 & الانتاج & |تربيعية & $r$ & \\
\hline$r 0.9$ & T.. & 7.9 & $\varepsilon 1$ & ص ص هـ & المساحة & خطية & $r$ & \multirow{2}{*}{ عباد الثمس } \\
\hline 49.8 & 71.0 & 7.0 & VI & 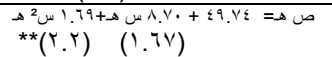 & الإنتاج & |تربيعية & $\varepsilon$ & \\
\hline 1.9 & TV. 1 & $\varepsilon .9$ & $\varepsilon V$ & ص هـ = & المساحة & خطبة & 。 & \\
\hline 9.1 & rq.r & $\varepsilon . r$ & $\leq 9$ & 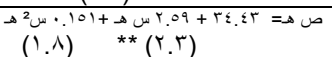 & الإنتاج & |تربيعية & 7 & \\
\hline
\end{tabular}

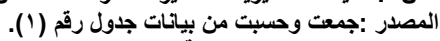

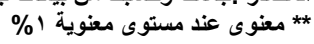

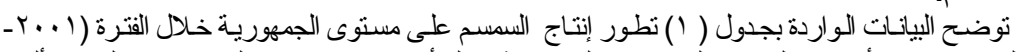

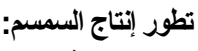

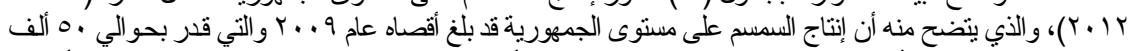

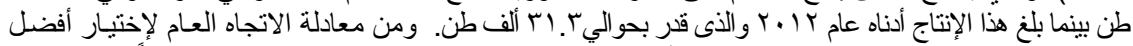

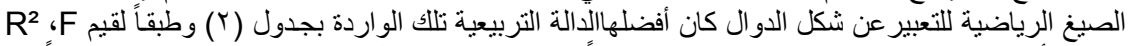

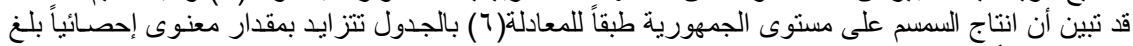

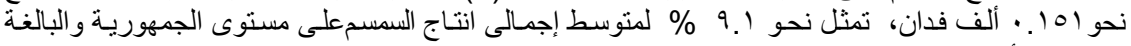

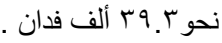
الاستهلاك المحلى من الفول السودان الفوانى:

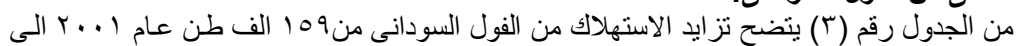

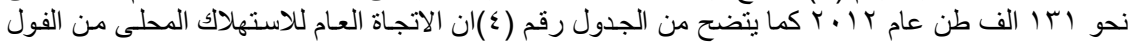

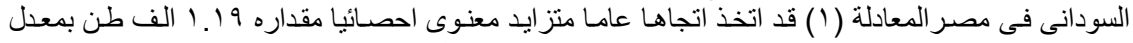

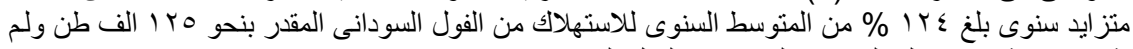

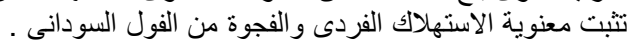




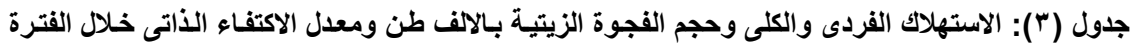

\begin{tabular}{|c|c|c|c|c|c|c|c|c|c|c|c|c|}
\hline السمسم & الشمس & سوداني & السمسم & الثبمس & سوداني & | السمسم | & الثمس & سوداني & | السمسم & الشمس & سودانى & \multirow[t]{2}{*}{ | للسنوات | } \\
\hline \multicolumn{3}{|c|}{ الفجوة الذيتية } & \multicolumn{3}{|c|}{ الاكتفاء الذاتىى\% } & \multicolumn{3}{|c|}{ الاستهلاك الفردى } & \multicolumn{3}{|c|}{ الاستتهلاك الكلى } & \\
\hline-89 & -18 & -94 & 28 & 71 & 14 & 1.9 & 2 & 1.7 & 124 & 62 & 109 & $r \ldots 1$ \\
\hline-45 & -9 & 70 & 45 & 80 & 158 & 1.2 & 0.6 & 1.8 & 82 & 44 & 121 & $r \ldots r$ \\
\hline-45 & -12 & -73 & 45 & 73 & 60 & 1.2 & 0.4 & 1.8 & 82 & 44 & 183 & $r \ldots r$ \\
\hline-38 & 18 & 78 & 49 & 169 & 169 & 1.1 & 0.4 & 1.7 & 75 & 26 & 113 & $r \ldots \varepsilon$ \\
\hline-7 & -99 & 93 & 84 & 23 & 188 & 0.6 & 1.8 & 1.5 & 44 & 129 & 106 & r...o \\
\hline-5 & -92 & 59 & 89 & 28 & 147 & 0.5 & 1.8 & 1.8 & 46 & 128 & 125 & $r \ldots 7$ \\
\hline-5 & -130 & 104 & 89 & 18 & 191 & 0.6 & 2.2 & 1.6 & 47 & 158 & 114 & $r \ldots v$ \\
\hline-18 & -92 & 72 & 67 & 18 & 153 & 0.7 & 1.5 & 1.8 & 54 & 112 & 137 & $r \ldots r$ \\
\hline-10 & -107 & 84 & 83 & 27 & 174 & 0.8 & 1.9 & 1.4 & 60 & 147 & 114 & $r \ldots q$ \\
\hline-14 & $\begin{array}{l}-83 \\
\end{array}$ & 88 & 77 & 44 & 177 & 0.8 & 1.9 & 1.4 & 60 & 147 & 114 & r.1. \\
\hline-2 & -112 & 75 & 96 & 14 & 157 & 0.6 & 1.7 & 1.7 & 45 & 130 & 132 & $r+11$ \\
\hline-14 & -118 & 74 & 69 & 14 & 156 & 0.6 & 1.7 & 1.7 & 45 & 138 & 131 & $r . \mid r$ \\
\hline-24 & -71 & 53 & 68 & 48 & 145 & 1 & 1 & 2 & 64 & 105 & 125 & المتو سط \\
\hline
\end{tabular}

الاستهلاك المحلى من عباد الثمس:

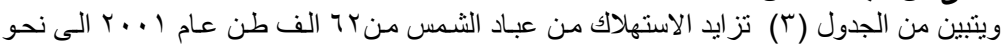

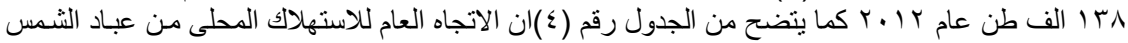

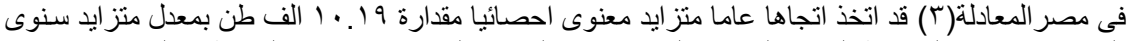

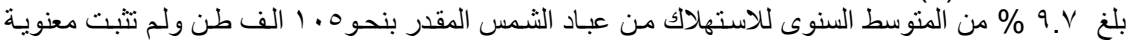
الاستهلاك الفردى بعباد الثمس.

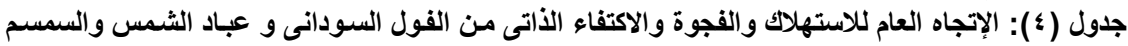

\begin{tabular}{|c|c|c|c|c|c|c|c|c|}
\hline & & & & & - 1-r. & لتترة () & خلال ال & \\
\hline 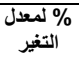 & المتوسط & j & ف & المعادلة & المتغير التابع & المعادلة & 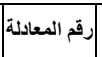 & المحصول \\
\hline$r . \varepsilon$ & iro & $r \varepsilon$ & r. & 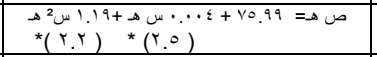 & استهلاك كلى & تربيعية & 1 & \multirow{2}{*}{ فول سودانى } \\
\hline $0 . \wedge$ & $1 \leqslant 0$ & 01 & $\because .1$ & 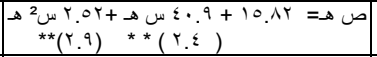 & اكتفاء ذاتى & تربيعية & r & \\
\hline $9 . \mathrm{V}$ & 1.0 & 71 & $10 . \varepsilon$ & $\begin{array}{l}\Delta \omega \\
* *(r .9)\end{array}$ & استهلاك كلى & خطية & $r$ & \multirow{3}{*}{ |عباد الثمس } \\
\hline $10 . \mathrm{V}$ & «^ & rv & $0 . \mathrm{V}$ & 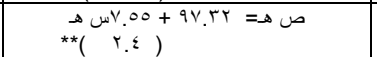 & اكتفاء ذاتى & خطية & $\varepsilon$ & \\
\hline $1 \leqslant . V$ & vi- & TV & $r \cdot r$ & 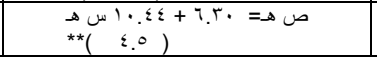 & فجوة غذائية & خطية & 。 & \\
\hline$v .7$ & $1 \varepsilon$ & $\infty 0$ & KY.Y & 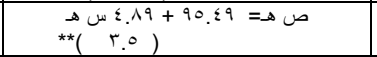 & استهلاك كلى & خطية & 7 & \multirow{4}{*}{ سمسم } \\
\hline$\Delta r$ & 1 & $0 \leqslant$ & 11.1 & 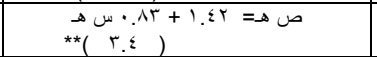 & استهلاك فردى & خطية & v & \\
\hline $7 . \varepsilon$ & $\pi$ & $0 \leqslant$ & 11 & 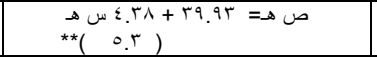 & اكتفاء ذاتى & خطية & $\wedge$ & \\
\hline$r .0$ & $r \varepsilon$ & or & $1 r . r$ & 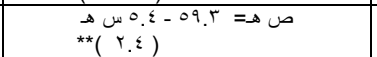 & فجوة غذائية & خطية & 9 & \\
\hline
\end{tabular}




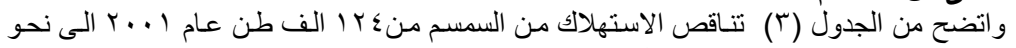

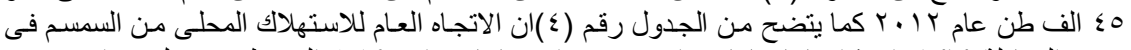

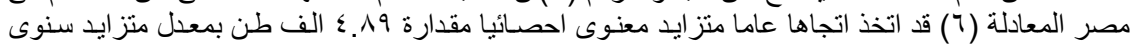

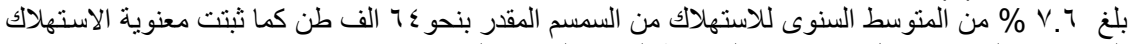

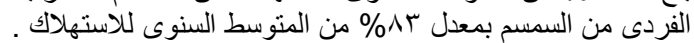

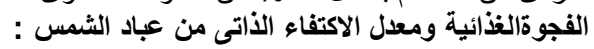

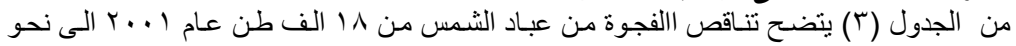

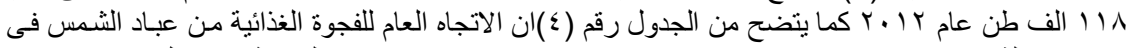

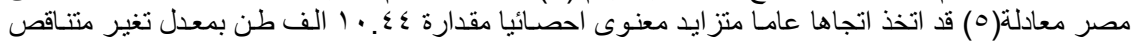

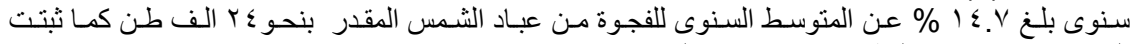

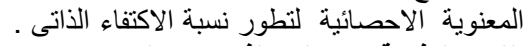

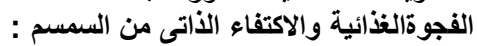

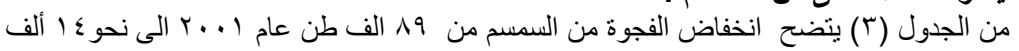

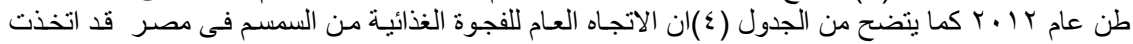

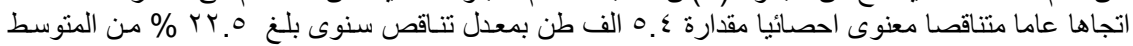

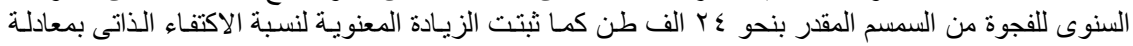

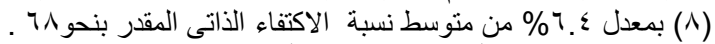

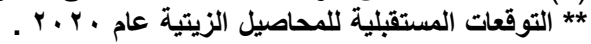

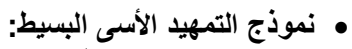

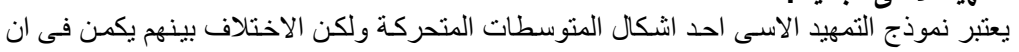

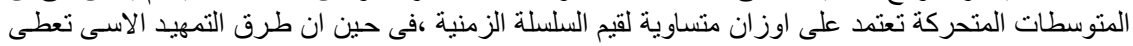

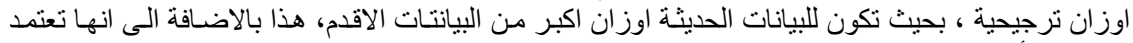

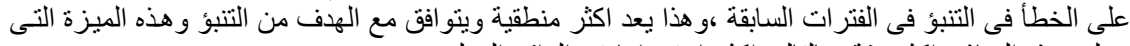

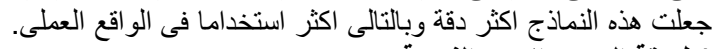
تعتمدهذه الطريقة فى التنبؤ على المعادلة الاتية :

$F_{(t+1)}=F_{(t)}+\alpha\left(y_{t}-F_{t}\right)$

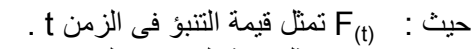

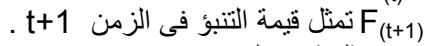

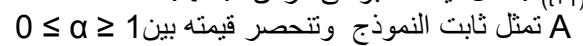

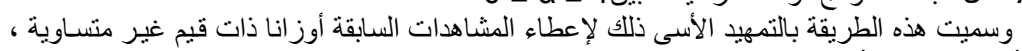

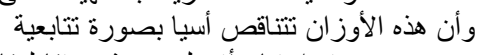

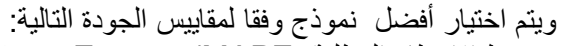

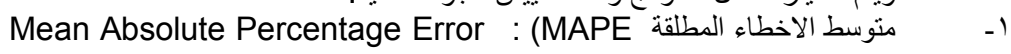

$$
\text { MAPE }=\frac{1}{n} \sum_{t=1}^{n}\left|\frac{y_{t}-\hat{y_{t}}}{y_{t}}\right|
$$

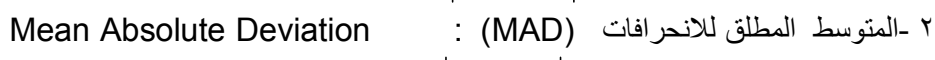

$$
\mathbf{M A D}=\frac{1}{n} \sum_{t=1}^{n}\left|y_{t}-\hat{y}_{t}\right|
$$




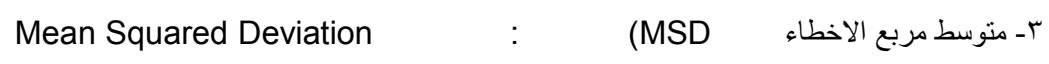

$\mathbf{M S D}=\frac{1}{n} \sum_{t=1}^{n}\left|y_{t}-\hat{y}_{t}\right|^{2}$

ويعتبر افضل نموذج هو النموذج الذى يحقق اقل انحر افات .

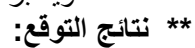

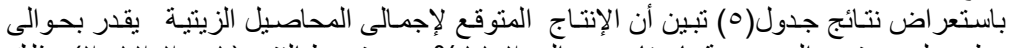

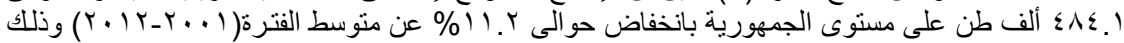

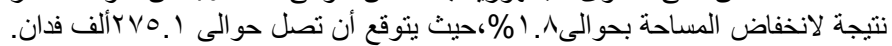

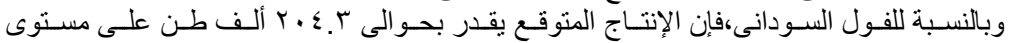

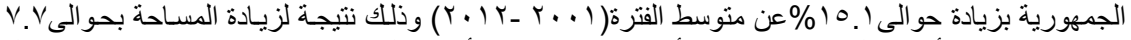

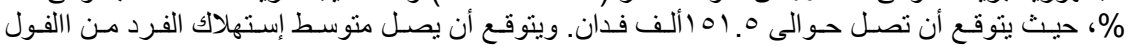

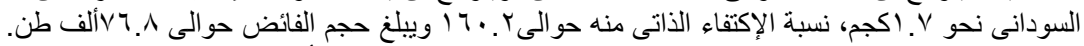

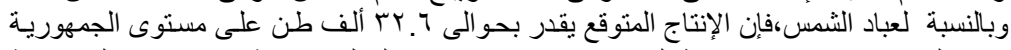

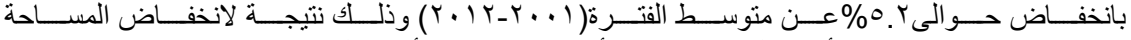

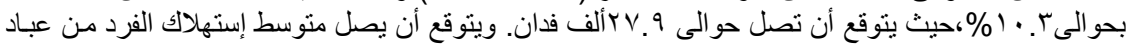

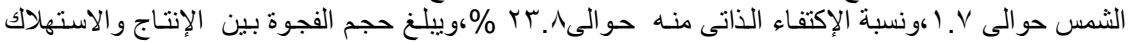

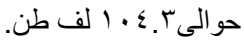

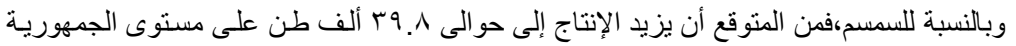

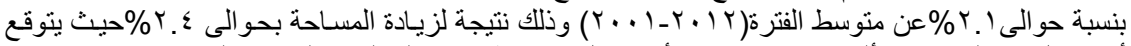

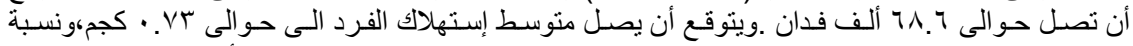

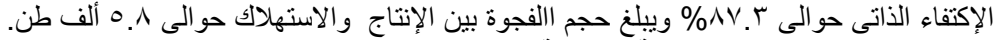

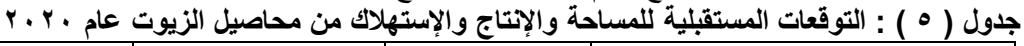

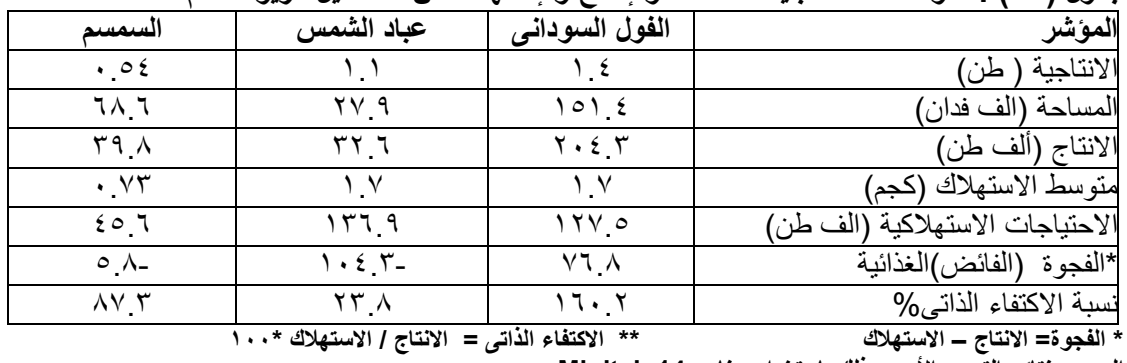
المصدر:نتائج التمهيد الأسىى وذلك باستخدام برنامج 14 Minitab.

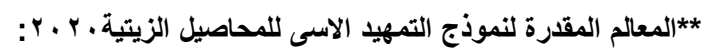

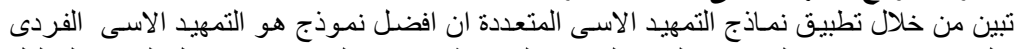

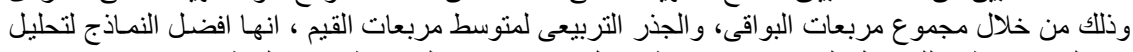

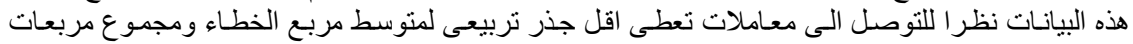

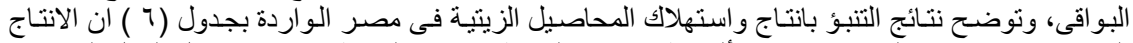

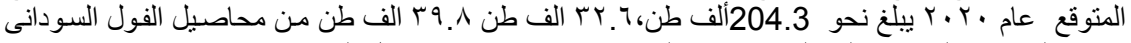

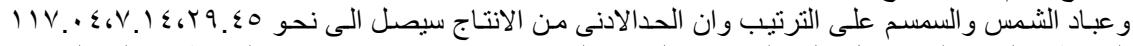

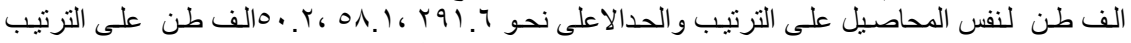

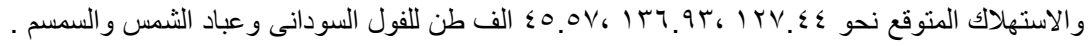


Fawzia A. Saber et al.

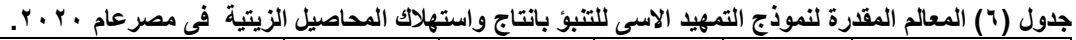

\begin{tabular}{|c|c|c|c|c|c|}
\hline $\begin{array}{l}\text { متوسط مربع الاخطاء } \\
\text { MSD }\end{array}$ & Forecast & Lower & Upper & $\alpha$ & المتغير \\
\hline & & & & & المحاصيل الزيتية \\
\hline & & & & & |الفول السودانى \\
\hline YNOY.Y & Y.乏.YY & $11 V_{.} \cdot \varepsilon$ & r91.09 &.$£ 07$ & |نتاج/ ألف طن \\
\hline$\varepsilon r \mu . \cdot \varepsilon$ & ITV. $\leqslant \varepsilon$ & 19.19 & 170.71 & $\because$. SYY & |ستهلاك /ألف طن \\
\hline & & & & & |عباد الشمس \\
\hline $1 V 0 . \leqslant 1$ & TY.TI & $V .1 \varepsilon$ & $01 . \cdot 1$ &. .119 & |انتاج/ ألف طن \\
\hline IY.V.乏. & 147.94 & $V V . .1$ & 197.17 &. .110 & |ستهلاك/ ألف طن \\
\hline & & & & & |السمسسم \\
\hline$r V . \wedge T$ & $r q . \wedge r$ & r9. 50 & $0 . r^{\prime}$ & $.1 r$. & |نتاج/ ألف طن \\
\hline Yo 0.71 & $\leqslant 0.0 \mathrm{~V}$ & $11 . r \Lambda$ & VY.VT & 1.19 & |ستهلاك/ ألف طن \\
\hline
\end{tabular}

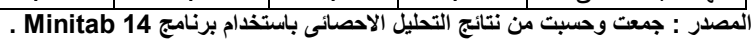

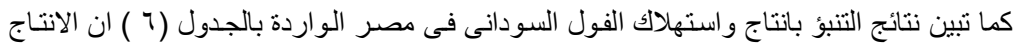

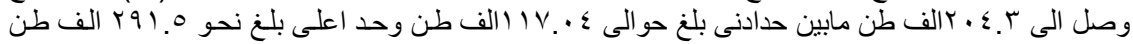

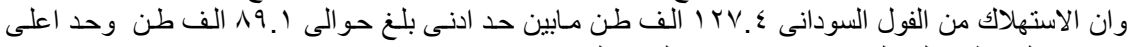

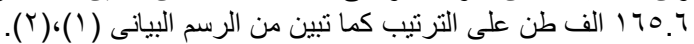

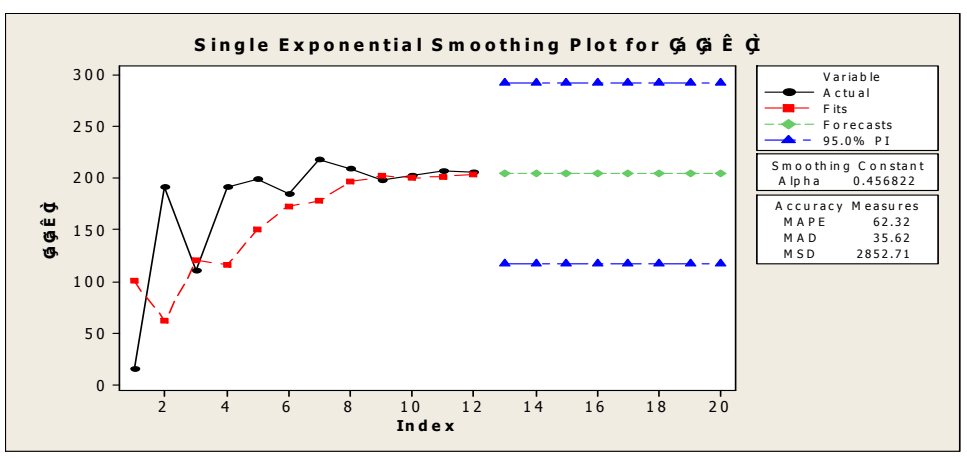

شكل بيانى ( ) كمية الا نتاج من الفول السودانى

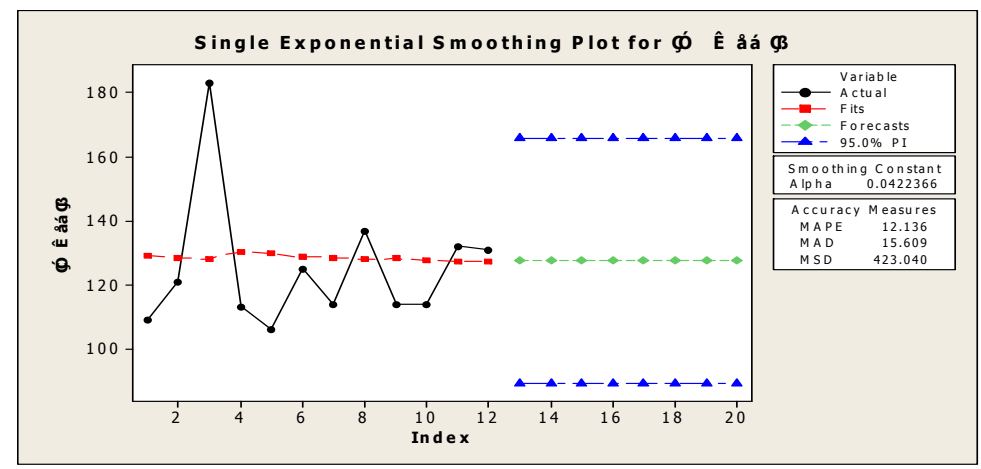

شكل بيانى (Y) كمية الا ستهلاك من الفول السودانى 


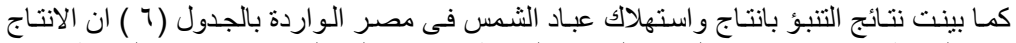

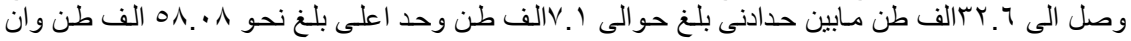

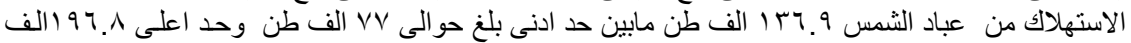

طن على الترتيب كما تبين من الرسم البيانى (r)،(ع ).

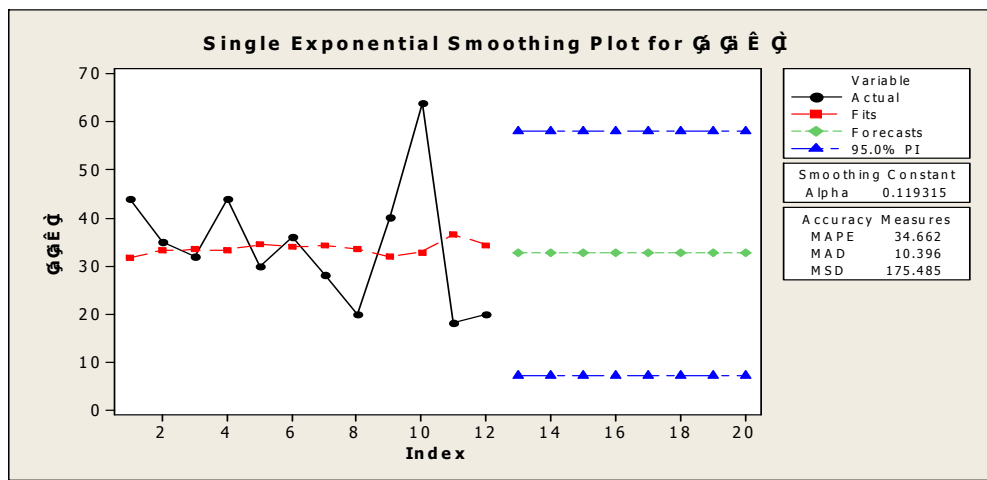

شكل بيانى (זr) كمية الا نتاج من عباد الشمس

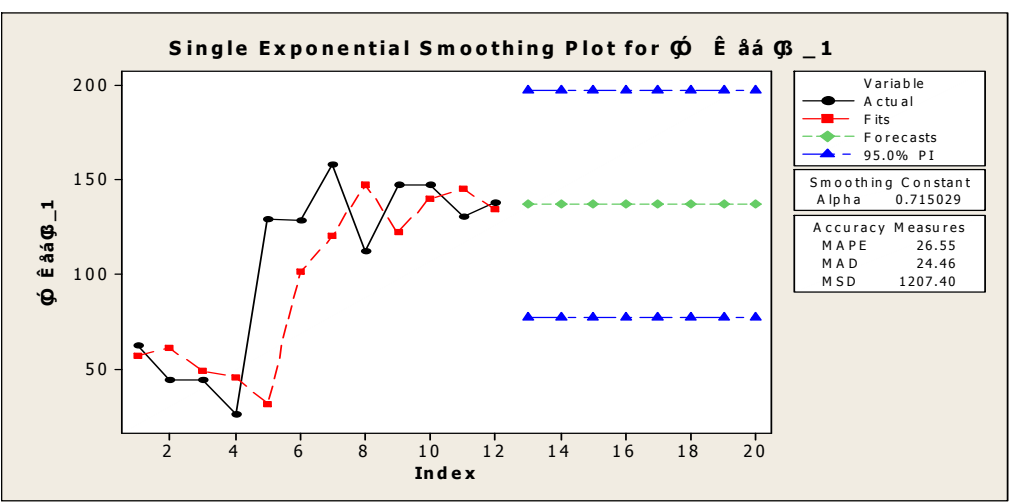

شكل بيانى (؛ ) كمية الا ستهلاك من عباد الشمس

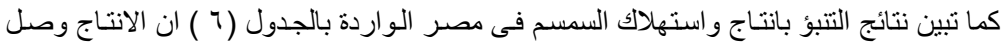

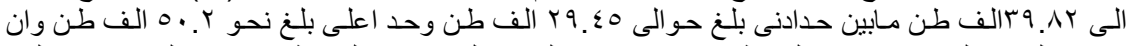

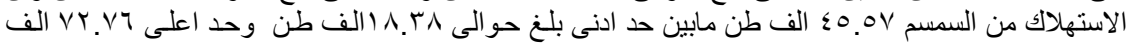

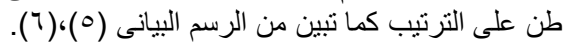


Fawzia A. Saber et al.

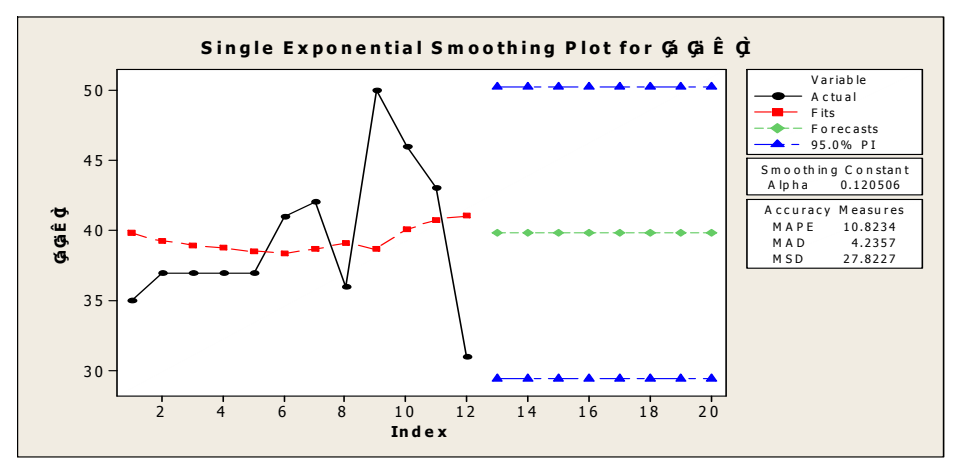

شكل بيانى (0) كمية الانتاج من سمسم

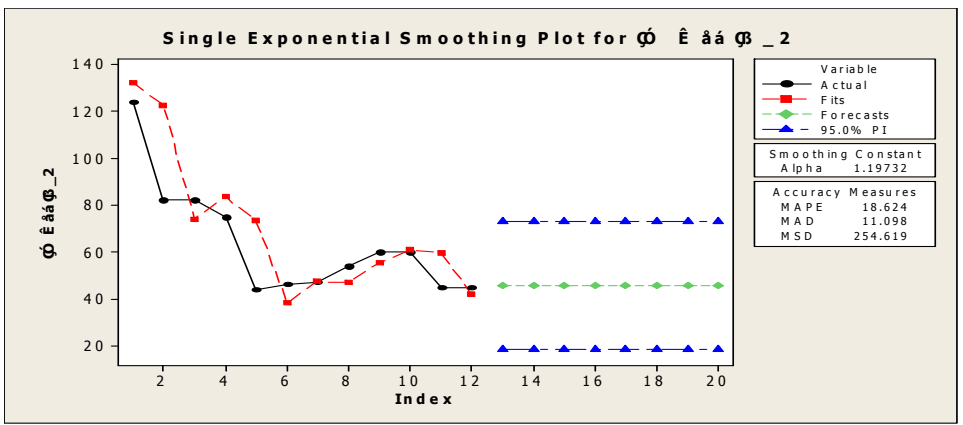

شكل بيانى (ך) كمية الا ستهلاك من السمسم

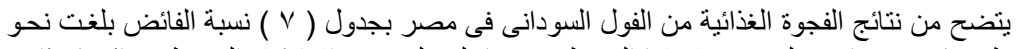

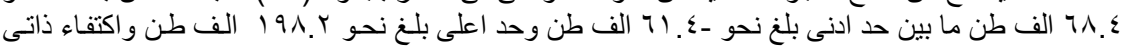

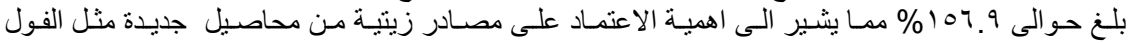

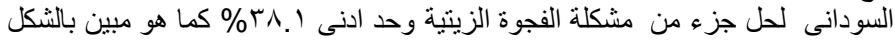

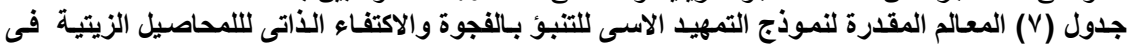

\begin{tabular}{|c|c|c|c|c|c|}
\hline & & & & \multicolumn{2}{|c|}{$r \cdot r$} \\
\hline $\begin{array}{c}\text { متوسط مربع الاخطاء } \\
\text { MSD }\end{array}$ & Forecast & Lower & Upper & $\alpha$ & |المتغير |لمير|| \\
\hline & & & & & |الفول السو دانى \\
\hline$r \leqslant 04.1$ & $7 \Lambda . \leqslant \varepsilon$ & $71 . \leqslant-$ & $191 . Y$ & 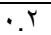 & |الفجوة/ ألف طن \\
\hline$r \wedge \lambda .0$ & 107.9 & rᄉ. & YVO.A &.$\wedge$ & الاكتفاء الذاتي\% \\
\hline & & & & & |عباد الثمس \\
\hline YITI. & $9 \cdot . \wedge-$ & IAY.Y. & $0 . \mathrm{V}$ & $\cdot . \mathrm{r}$ & |الفجوة / ألف طن \\
\hline 1919.8 & $r \varepsilon .1 V$ & $\sum V_{.} \Lambda_{-}$ & 117.4 & $\cdot . \mathrm{Y}$ & الاكتفاء الذاتي\% \\
\hline & & & & & |السمسسم \\
\hline $0 \leqslant 0.9$ & 10.19 & $09 . \Gamma \Lambda_{-}$ & TY.O & $\cdot . \mathrm{r}$ & |الفجوة / ألف طن \\
\hline$\xi . Y_{0}{ }^{2}$ & VO.YY & r..V & $11 r . V$ & $\cdot . \mathrm{Y}$ & الاكتفاءالذاتي\%\% \\
\hline
\end{tabular}


J. Agric. Econom. and Social Sci., Mansoura Univ., Vol.6 (4), April ,2015

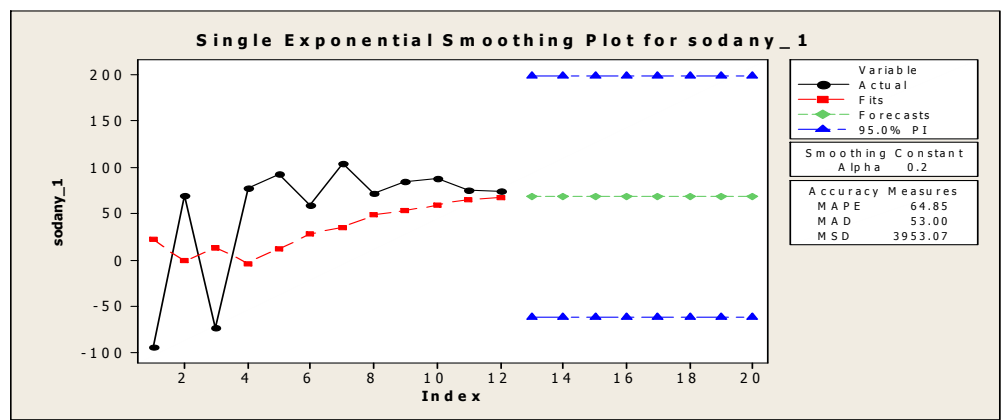

شكل بيانى (V) الفجوة من الفول السودانى

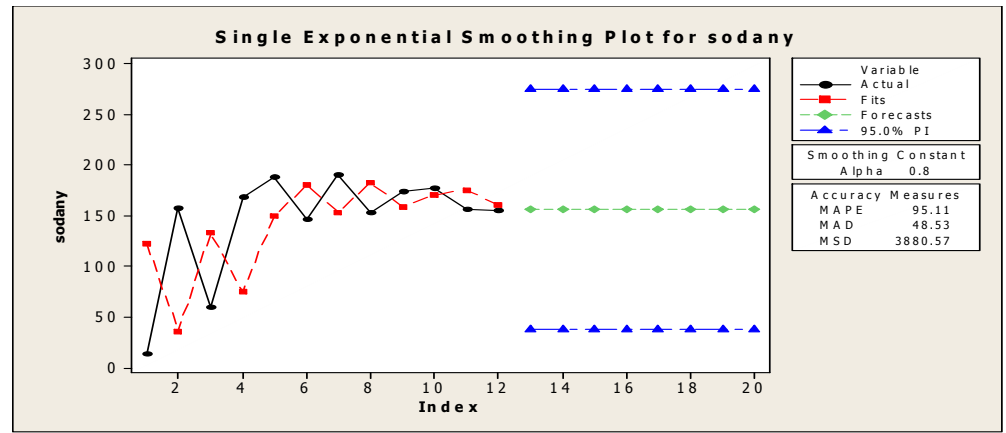

شكل بيانى (^) الاكتفاء الأتى من الفول السودانى

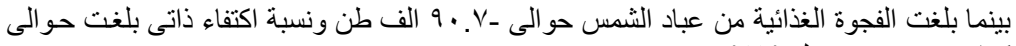

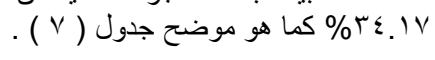

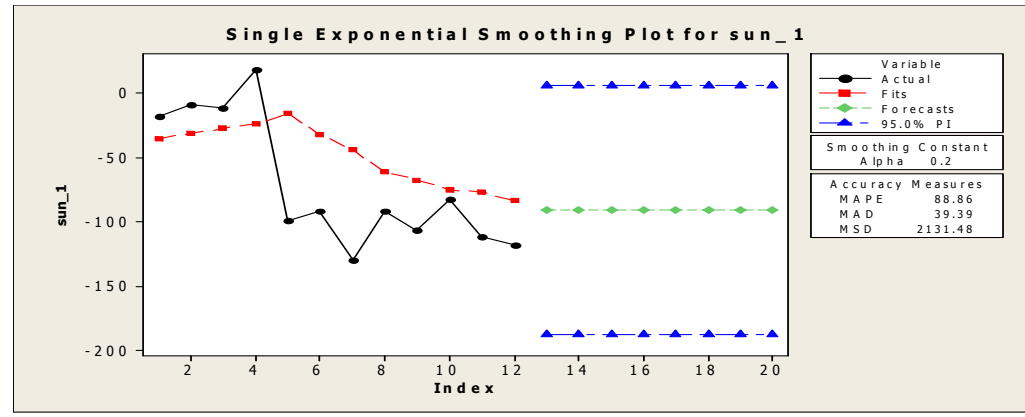

شكل بيانى (9) الفجوة من عباد الشمس 
Fawzia A. Saber et al.

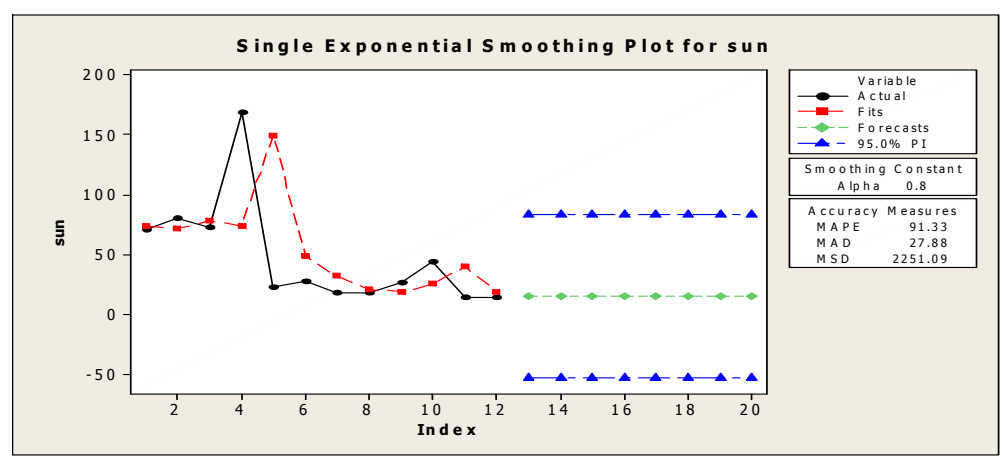

شكل بيانى ( • (1) الاكتفاء الأتى من عباد الشمس

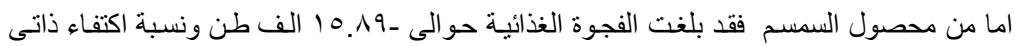

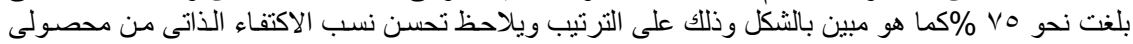

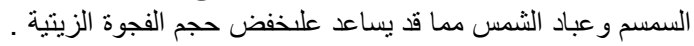

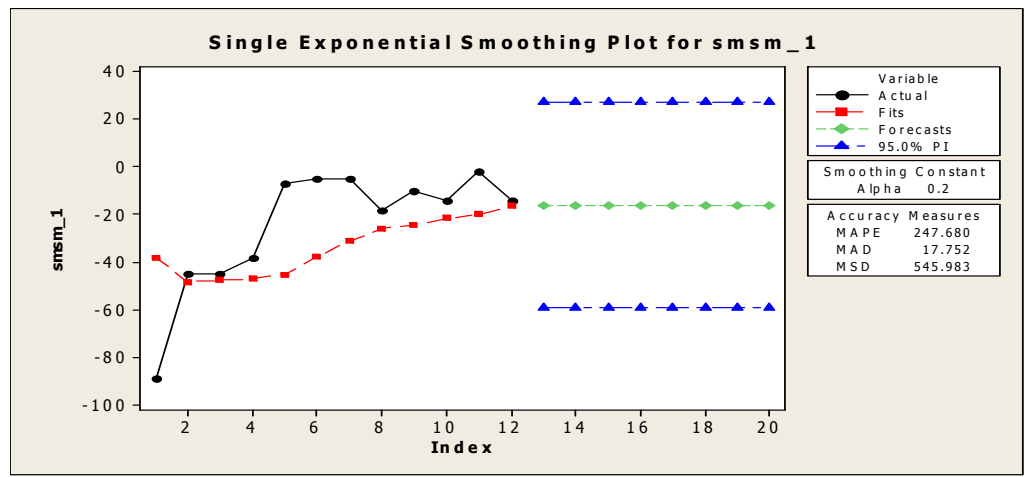

شكل بيانى (1 1) ) الفجوة من السمسم

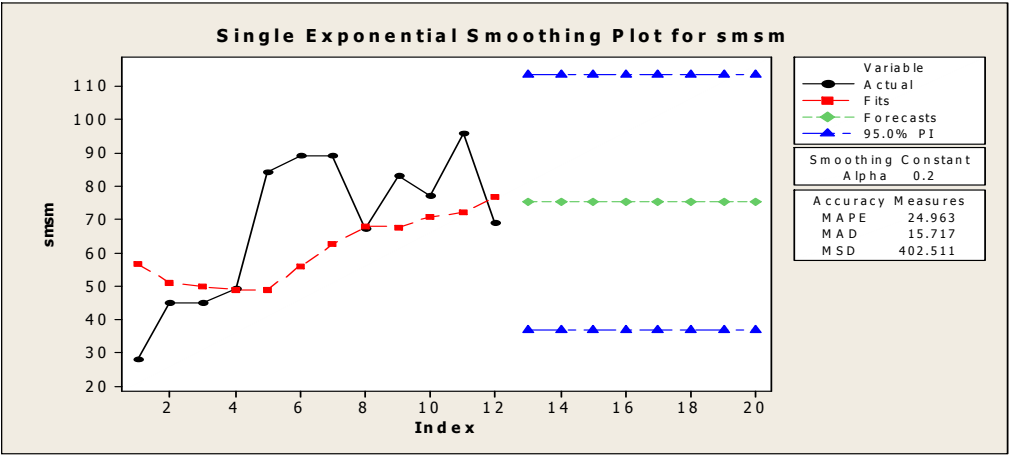

شكل بيانى (r l ) الاكتفاء الذاتى من السمم 


\section{المراجع}

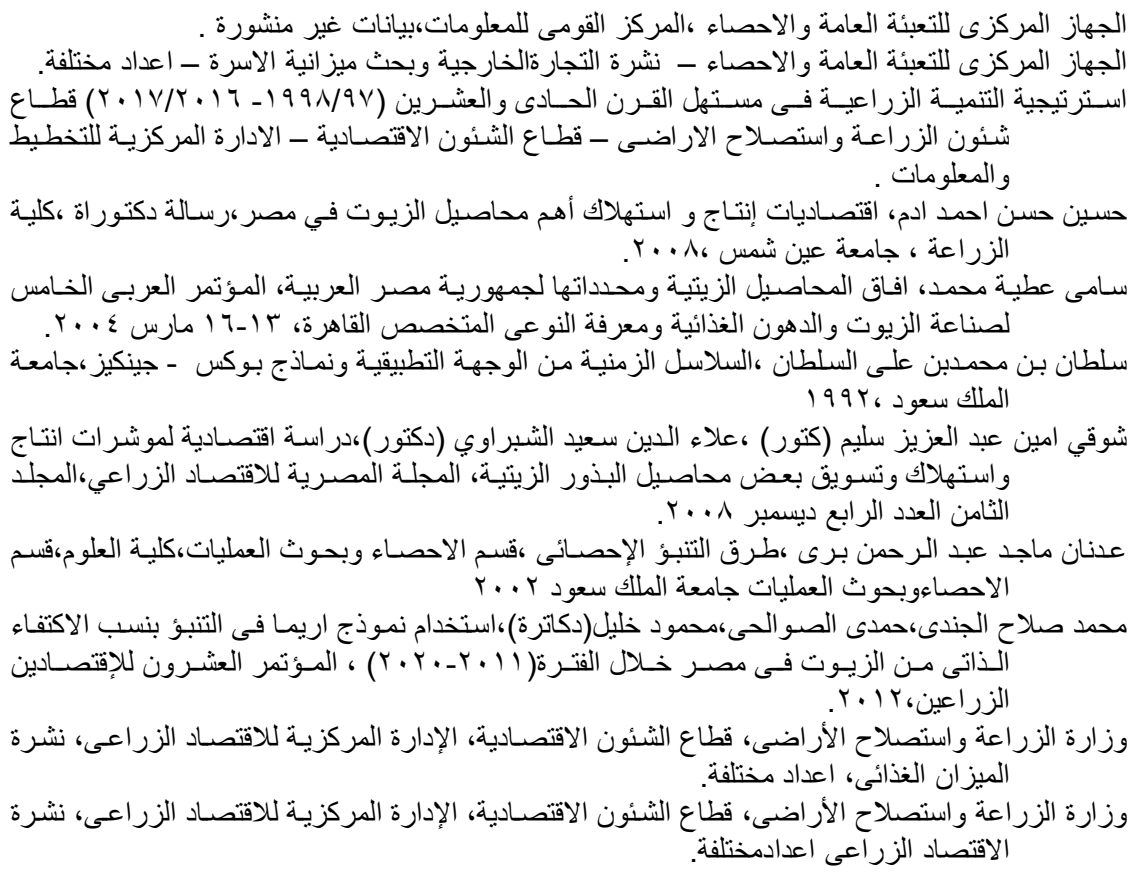




\section{AN ECONOMIC STUDY OF THE MOST IMPORTANT OIL CROPS IN EGYPT

\author{
Fawzia A. Saber ; AZZA M. Ghazala and Samar M. Alkady \\ Department of Economic Studies - Division of Economic and Social \\ Studies - Desert search Center - Cairo - Egypt.
}

\section{ABSTRACT}

Oil Crops are important strategic crops in Egypt or in countries around the world, it considered a major source of food in terms of human consumed in different ways in the diet is also important food commodities which the food gap, where up Self-sufficiency ratio of vegetable oils in the range of 10 from $13 \%$ despite the multiplicity of oil crops that can be grown in Egypt, but the limited space of this crop does not exceed $1.7 \%$ of the cropped area in Egypt, The problem with search that despite increase in domestic production, but there is still a gap between the production and consumption of oil crops, which form a burden on the Egyptian trade balance so targeted research identify the development of the production and consumption of the most important oil crops in Egypt and self-sufficiency of crops under study The results obtained from the results of future projections indicate that expected from crop peanuts lost production arrived and consumption respectively about 204 thousand tons, 127 thousand tons, either for production sunflower crop was expected output reached about 33 thousand tones and consumption to 137 thousand tons and crop sesame to about 39 thousand tons and consumption to 46 thousand tons, and appreciated oily gap and the rate of self-sufficiency of crops under study has oily gap to harvest groundnuts amounted to about 68 thousand tons and the percentage of sufficiency of about $160 \%$. As for the crop sunflower was the gap and the ratio sufficiency self on the order of about 90 thousand tons of $0.34 \%$ and reached oily gap to harvest sesame to about 16 thousand tons and Self-sufficiency ratio to about $75 \%$, which indicates deems necessary direction to new varieties and new sources of oil to fill the oil gap which make commitment concerted efforts to raise the oil crops,

Finally, the study found the possibility of to improve the self-sufficiency of oils and reduce the future size of the oily gap using the resources available and dependence on the types of new varieties of crops and government intervention develop policies that encourage the cultivation and production of oil crops such as groundnuts, sesame and the creation of good contemporary of these crops and to review the crop composition to include non-traditional crops to produce oils The study recommends.

1- develop a strategy and goals for the advancement of the oily crops include planting new crops paintings relied upon to fill the oil gap.

2- Work on high-quality production from varieties of peanut and sesame crops cultivation.

3. The introduction of other varieties of oil unconventional highly productive crops bear different climatic conditions in land cultivated, such as the cultivation of peanuts New Territories.

4- use extension tools to connect with farmers to guide them with new varieties with high productivities.

5. Work on raising awareness to rationalize consumption and reduce waste oils. 